\title{
Implementation of a Broad Range Smart Temperature Measurement System Using an Auto-Selecting Multi- Sensor Core in LabVIEW
}

\author{
Sidi M. Ahmed Ghaly \\ Electrical Engineering Department, \\ College of Engineering, Al Imam \\ Mohammad Ibn Saud Islamic University \\ (IMSIU), Riyadh, Saudi Arabia \\ smghaly@imamu.edu.sa \\ Mohammed Al-Awad \\ Electrical Engineering Department, \\ College of Engineering, Al Imam \\ Mohammad Ibn Saud Islamic University \\ (IMSIU), Riyadh, Saudi Arabia \\ maawaad@imamu.edu.sa
}

\author{
Mohammad Obaidullah Khan \\ Electrical Engineering Department, \\ College of Engineering, Al Imam \\ Mohammad Ibn Saud Islamic University \\ (IMSIU), Riyadh, Saudi Arabia \\ OKKhan@imamu.edu.sa \\ Mohammad Asad Ali \\ Electrical Engineering Department, \\ College of Engineering, Al Imam \\ Mohammad Ibn Saud Islamic University \\ (IMSIU), Riyadh, Saudi Arabia \\ asadali@imamu.edu.sa
}

\author{
Souleimane Ould El Mehdi \\ Electrical Engineering Department, \\ Ecole Normale Superieure \\ Mauritanie, \\ Nouakchott, Mauritania \\ souleiman6@ens.mr \\ Khalid Al-Snaie \\ Electrical Engineering Department, \\ College of Engineering, Al Imam \\ Mohammad Ibn Saud Islamic University \\ (IMSIU), Riyadh, Saudi Arabia \\ kalsnaie@imamu.edu.sa
}

\begin{abstract}
A conventional temperature sensing system employs a single transducer to convert temperature into an electrical signal. Such a system suffers from the limitation of the sensing range of the sensing device thereby affecting its accuracy and its capability. Therefore to make an accurate measurement in a typically abrupt temperature varying environment, a broad range high precision sensor is required. In this paper, an attempt is made to implement a wide spectrum temperature measurement system using auto-selected multi-sensor core in LabVIEW. This multi-sensor core can be composed of a set of different sensors having different capabilities to measure different temperatures ranges. These sensors are auto-selected by the program depending on the environment. This concept may be useful for space applications or it can also be useful for the monitoring of temperature and pressure in an oil/gas transportation or supply by means of underground/sea pipeline system or in a refinery plant. Further, this may also be applied for high precision temperature sensing in magnetic resonance imaging system applications.
\end{abstract}

Keywords-broad spectrum temperature range measurement; multi-sensor core; LM-35; thermister; RTD; auto-select sensor; linearization; LabVIEW

\section{INTRODUCTION}

Temperature measurement is carried out by various devices, each with its own limitations of operating temperature range and capabilities. The purpose of this paper is to develop an idea and technique by which temperature measurement can be achieved through a varying wide range system. For this intention, three different types of sensors having three different temperatures ranges were employed. The three sensors are thermistor, Resistance Temperature Detector (RTD) and LM35 sensor [1-4]. These three sensors are connected to a logic circuit which selects one particular sensor at a time. A software simulation program is developed such that at the start of the measurement a reference temperature and corresponding reference sensor are chosen. As the temperature increases, the software simulation program checks the temperature and the sensors' range, a suitable sensor is selected among the set, and the system measures the temperature from the selected sensor as long as the range provided by the program is met. Once the range crosses the threshold value, the software simulation program will check for another suitable sensor and so on. By this design the instrument measures varying temperatures by changing sensors. A similar approach is employed for decreasing temperatures. The sensors which are not used at the time of measurement should be kept switched-off so that higher temperatures do not damage unused sensors. At the same time the system does not give erroneous readings when its output is taken by a summing amplifier. The selection of sensors can be achieved by using a switching logic circuit connected to the set of sensors. These sensors give output in terms of voltage that corresponds to the measured temperature. The developed concept can be useful for many industrial applications, especially in magnetic imaging systems [5-8].

\section{IMPEMENTATION OF A MULTI-RANGE SENSING SYSTEM}

A multi-range sensing system is established as illustrated in Figure 1. To implement this idea, three linearized resistor sensors, namely thermistor, RTD, and an integrated circuit sensor LM-35 are used. Based on the environmental temperature range, the sensor auto-selecting circuit selects one 
of the three sensors to measure the sampled area temperature. The linearity [9] of these sensors is illustrated in the next section.

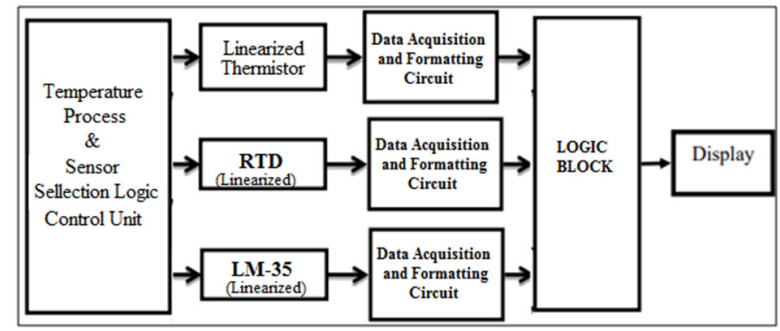

Fig. 1. Block diagram of the multi-range sensing system

Individual data acquisition and formatting circuits convert the received temperature signal, and based on individual underlying principles of conversions defined in (1) to (6), the temperature is measured and displayed. The principle of working and the linearization of each of the sensors are discussed below. Ideally, it is often desired that a strictly linear relationship exists between the input and output signals in these sensing devices. Practical units, however, will always have some degree of nonlinearity. If an instrument block has constant gain for all input signal levels, then the relationship response graphing the input against the output will be a straight line and the relationship is linear. The broad spectrum temperature measurement system using auto-selected multisensor core has been implemented and simulated in LabVIEW. LabVIEW is a graphical programming language which uses a graphical user interface. It is simple and user friendly. A user can select a block from its vast function library and define parameters, inputs, and outputs to model a particular system. Subprograms can be defined as sub-VIs. These sub-VIs can be connected, added, removed, adjusted or modified, modeled, tested, and re-modeled according to task parameter changes with time and requirements. LabVIEW, apart from providing programming, can easily be integrated to direct hardware without any interface programming or special requirements by using the DAC (data acquiring and controlling) assistant to different interfacing boards of National Instruments [10-11].

\section{A. The Thermistor}

Thermistors are temperature sensitive passive semiconductors, whose resistance can increase and decrease with temperature. The resistances vary by a large amount for small temperature changes. There are two types of thermistors: positive temperature coefficient (PTC) thermistor for which the resistance increases with increase in temperature, and negative temperature coefficient (NTC) thermistor for which the resistance decreases with increase in temperature. An NTC thermistor is used in temperature measurement [12]. Since the thermistor has non-linearity for temperature measurement versus measured resistance $R_{T}$, there should be some sort of linearization. For linearization to be obtained for our required range in a simple form we used two resistors $R_{1}$ and $R_{2} . R_{2}$ and $R_{T}$ are connected in parallel whereas $R_{1}$ is connected in series with the combination of $R_{2}$ and $R_{T}$. If a voltage of $V_{i n}$ is applied to the circuit as shown in Figure 2, the voltage across $R_{T}$ is given by $V_{\text {out }}$ :

$$
R_{T}=\frac{R_{2} R_{1}}{\frac{V_{\text {in }}}{V_{\text {out }}} R_{2}-\left(R_{2}+R_{1}\right)}
$$

Equation (1) gives the relation for the determination of $R_{T}$ from the applied input voltage $V_{i n}$, which is $1 \mathrm{~V}$, the output voltage measured which is $0.56 \mathrm{~V}$ to $0.78 \mathrm{~V}, R_{2}$ which is $15000 \Omega$ and $R_{l}$ which is $2350 \Omega$.

$$
T=\left[\frac{1}{T_{R}}+\frac{1}{B} \ln \left(\frac{R_{t}}{R_{r}}\right)\right]^{-1}
$$

The linearized NTC thermistor output Voltage $V_{\text {out }}$ versus temperature plot is illustrated in Figure 3.

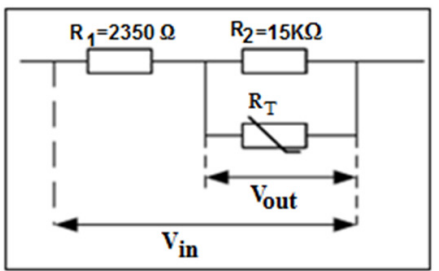

Fig. 2. NTC thermistor with input voltage Vin

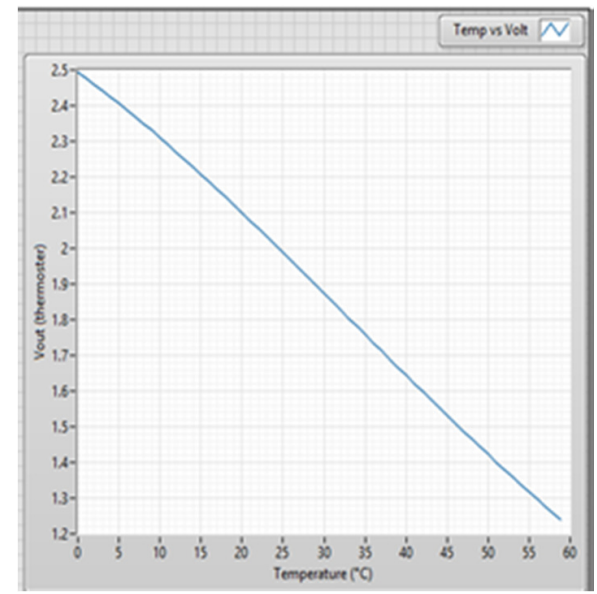

Fig. 3. Thermistor output Voltage $V_{\text {out }}$ versus measured temperature

Equation (2) gives the relation between the measured absolute temperature $T$, reference absolute temperature $T_{r}$ which is $298 \mathrm{~K}$ corresponding to the reference resistance $R_{r}$ which is $2252 \Omega$, obtained resistance $R_{T}$ from (1) and a parameter $B$ which is 1800 , corresponding to (2) and (1). This thermistor with a typically given range from $0^{\circ} \mathrm{C}$ to $60^{\circ} \mathrm{C}$ is used to measure temperature. However, it can be changed to any other range e.g. from $-50^{\circ} \mathrm{C}$ to $100^{\circ} \mathrm{C}$.

\section{B. Resistance Temperature Detector (RTD) Sensor}

RTDs are devices whose resistance increases with increase in temperature. These devices exhibit linearity response for temperature to Voltage (resistance) measurement. RTDs are used to measure metal temperature with high melting points and are able to stand corrosion. RTDs have high accuracy, but low change in resistance for temperature change [13, 14]. Therefore, they can be used to sense certain big levels of 
changes. Figure 4 shows an input voltage $V_{\text {in }}$ applied and an output (measured) voltage $V_{\text {out }}$.

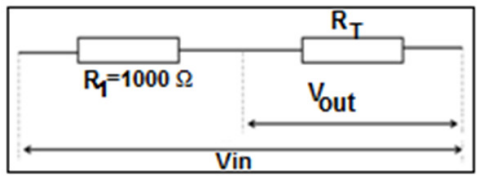

Fig. 4. RTD sensor

$$
\begin{aligned}
& R_{T}=\frac{V_{\text {out }} R_{1}}{V_{\text {in }}-V_{\text {out }}} \\
& T=\left[\frac{\left(\frac{R_{T}}{R_{r}}-1\right)}{\alpha}+T_{r}\right]
\end{aligned}
$$

Equation (4) gives the measured resistance $R_{T}$ from the input voltage $V_{\text {in }}$ which is $1 \mathrm{~V}$, output voltage $V_{\text {out }}$ which ranges from $0.12 \mathrm{~V}$ to $0.23 \mathrm{~V}$ for a range of $100 \Omega$ to $500 \Omega$ and resistance $R_{I}$ which is $1000 \Omega$. Equation (5) gives the absolute temperature measured from $R_{T}$ by (4) and reference resistance $R_{r}$ which is $100 \Omega$ for reference absolute temperature $T_{r}$ which is $273 \mathrm{~K}$ and a parameter called alpha $(\alpha)$ which is $0.0385^{\circ} \mathrm{C}^{-1}$. A typical LabVIEW frame to measure temperature with RTD sensor under linearization environment is shown in Figure 5. The linearized output voltage curve with respect to temperature is shown in Figure 6. Figure 7 shows the comparative study of linear and non-linear output Voltage with respect to measuring temperature for RTD in the range from $0^{\circ} \mathrm{C}$ to $850^{\circ} \mathrm{C}$.

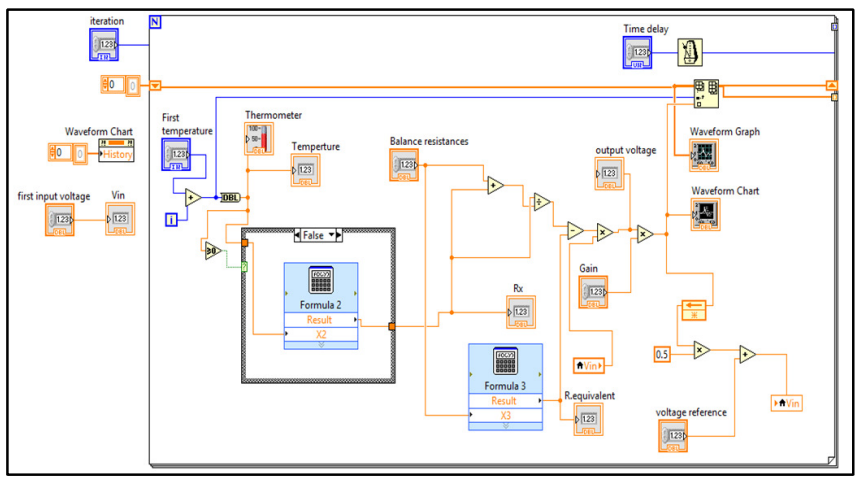

Fig. 5. LabVIEW frame for temperature measurement with RTD sensor under linearization

\section{The LM-35 Sensor}

The LM35 is an integrated circuit sensor that can be used to measure temperature with an electrical output proportional to temperature (in ${ }^{\circ} \mathrm{C}$ ). It can measure temperature more accurately than a thermistor. The sensor circuitry is sealed and not subject to oxidation. The LM35 generates a higher output Voltage than thermocouples and may not require the output Voltage to be amplified. The LM35 has an output voltage proportional to Celsius temperature. The scale factor is $0.01 \mathrm{~V} /{ }^{\circ} \mathrm{C}$. The LM35 does not require any external calibration or trimming. It maintains an accuracy of $\pm 0.4^{\circ} \mathrm{C}$ at room temperature and $\pm 0.8^{\circ} \mathrm{C}$ in the range of $0^{\circ} \mathrm{C}$ to $+100^{\circ} \mathrm{C}$. Another important characteristic of the LM35 is that it draws only $60 \mu \mathrm{A}$ from the supply and possesses a low self-heating capability.

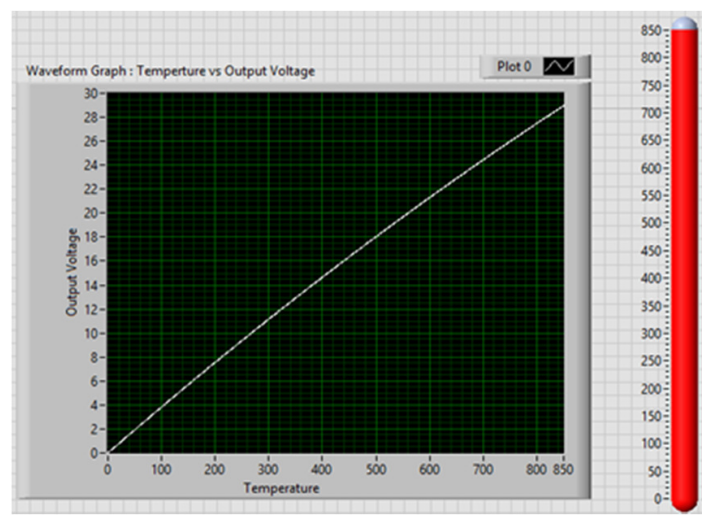

Fig. 6. RTD output Voltage $V_{\text {out }}$ versus temperature

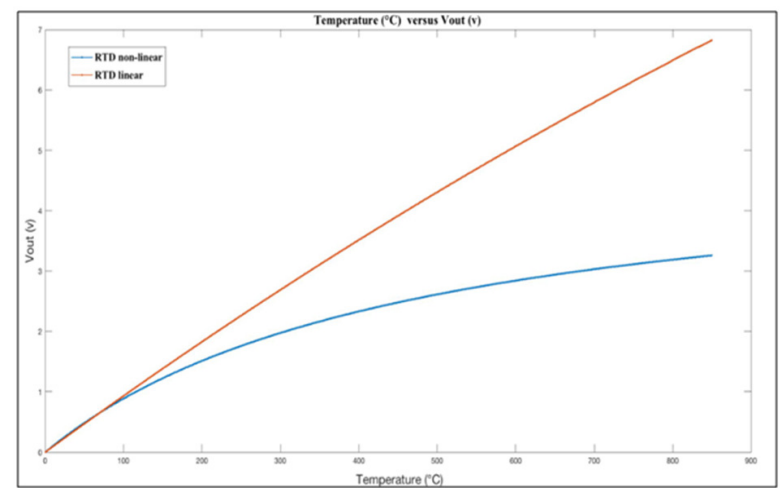

Fig. 7. Illustration of output Voltage response of RTD under linear and non-linear condition for temperature range of $0^{\circ} \mathrm{C}$ to $850^{\circ} \mathrm{C}$

The LM35 comes in many different packages and can be selected accordingly. This sensor allows the temperature between $-55^{\circ} \mathrm{C}$ to $150^{\circ} \mathrm{C}$ [15]. There are two transistors $\mathrm{Q}_{1}$ and $\mathrm{Q}_{2}$ in the center of the circuit. $\mathrm{Q}_{1}$ has ten times the emitter area of the other. This means it has one tenth of the current density, since the same current is going through both transistors. This causes a voltage across the resistor $R_{l}$ that is proportional to the absolute temperature, and is almost linear across the range. The "almost" part is taken care of by a special circuit that straightens out the slightly curved response of Voltage versus temperature. An amplifier ensures that the voltage at the base of transistor $\mathrm{Q}_{1}$ is proportional to the absolute temperature by comparing the output of the two transistors. Another amplifier converts absolute temperature (measured in Kelvin) into either Fahrenheit or Celsius, depending on the model (LM34 or LM35). This works according to (6):

$$
V_{\text {out }}=\frac{\text { Temperature in }{ }^{\circ} \mathrm{C}}{100}
$$

A typical linearity curve obtained from the temperature measurement system using LM-35 in LabVIEW environment is shown in Figure 8. A typical LabVIEW front panel display showing the selected sensor and a typical virtual temperature scale for temperature measurement is depicted in Figure 9. 


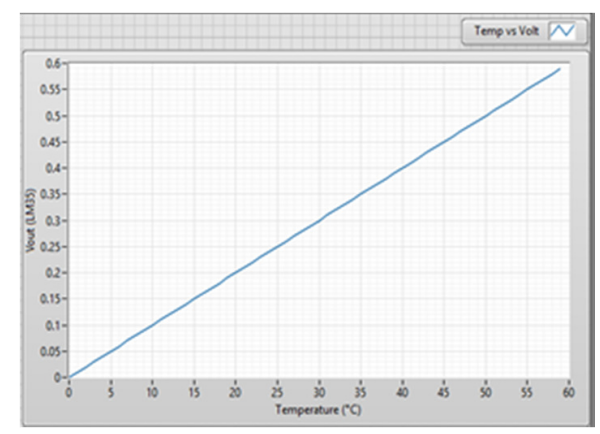

Fig. 8. LM35 temperature sensor linearization curve

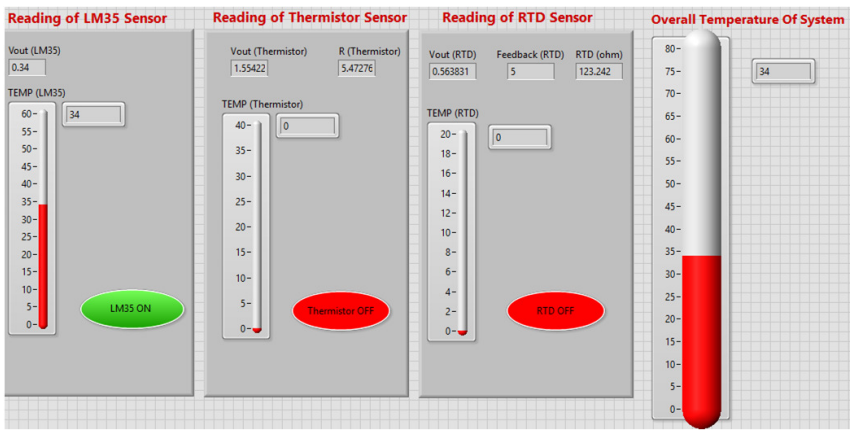

Fig. 9. LabVIEW front panel showing the selected sensor for temperature measurement.

\section{LABVIEW SIMULATIONS}

The individual LabVIEW frames were combined and worked as a single system based on a multi-range temperature measuring device in which each sensor is auto-selected by the underlying logic $[16,17]$. Figure 10 shows a combination of the three discussed above sensors with a logic circuit. The logic circuit has three inputs, one for each sensor and two selection switches (for manual selection) to select any sensor among the three. The signal output is obtained from the logic block, a software simulation program selects an initial sensor, the temperature is measured from that sensor and the software is programmed to select the sensor automatically as the temperature reaches the predefined sensor range. The software keeps the values of two previously measured temperatures in order to select upward or downward temperature measurement and then selects the appropriate sensor automatically. As the temperature increases or decreases and goes out of the range of the present sensor and reaches the range of another sensor, the other sensor is selected by the auto-selection switches of the logic circuit which are shown with their temperature ranges in the plot [18].

\section{RESUltS AND DISCUSSION}

The range of each sensor is assigned and the simulation is conducted in LabVIEW. A combined result is plotted and illustrated by using different colors. Typically LM-35 is assigned the temperature range of $-50^{\circ} \mathrm{C}$ to $100^{\circ} \mathrm{C}$, the thermistor is assigned the range of $101^{\circ} \mathrm{C}$ to $650^{\circ} \mathrm{C}$, and the RTD sensor is assigned the range of $651^{\circ} \mathrm{C}$ to $850^{\circ} \mathrm{C}$. Simulation experiments are repeated by changing the range of each sensor and it was noted that for a broad temperature range the simulation setup exhibited satisfactory results and the different sensors were selected automatically to operate in their assigned ranges, as illustrated in Figure 11. Thus, using the proposed method, this devised instrument can measure the temperature from a range of $-50^{\circ} \mathrm{C}$ to around $1000^{\circ} \mathrm{C}$. A sufficient number of simulations were carried out by changing temperature ranges assigned to each sensor and the results were preserved. The combined results of this technique are illustrated in Figure 11.

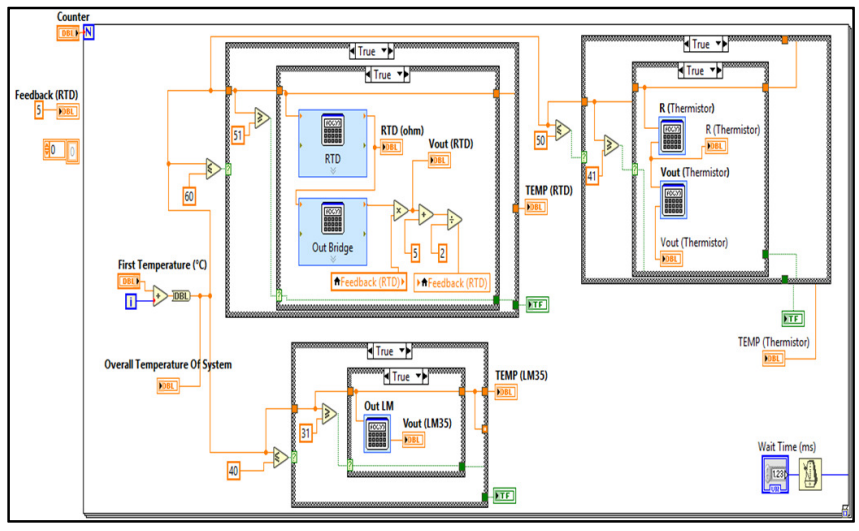

Fig. 10. LabVIEW frame of the block diagram of multi-range sensing

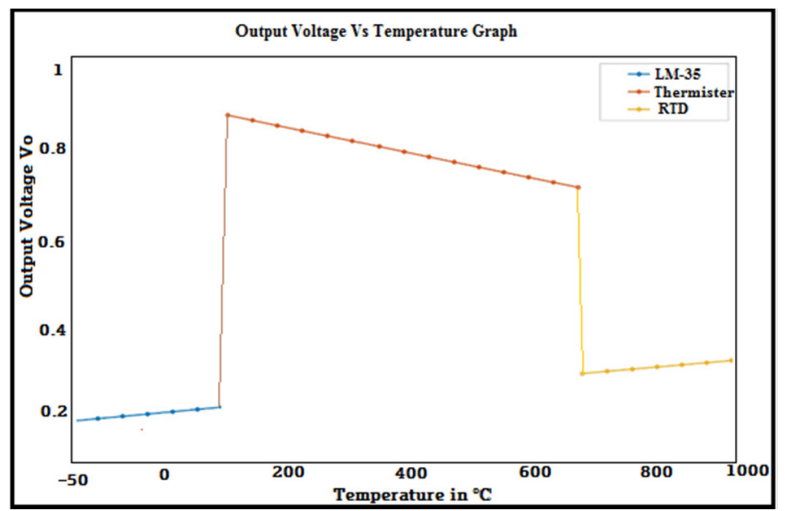

Fig. 11. Color illustration of the total temperature range measured by individual sensors

\section{CONCLUSION AND FUTURE WORK}

The proposed mechanism was devised with the help of LabVIEW. For a broad temperature range, the technique of using a multi-sensor auto-selecting system with the help of a software program for increasing the range of temperature measurement was employed and simulated. The combination of sensors can be used to display various physical quantities like temperature, air pressure and humidity in weather prediction. The future scope of this mechanism is to be employed for the monitoring of temperature and pressure in oil or gas transportation or supply by means of underground/sea pipeline system or in a refinery plant. Furthermore, this may also be applied for high precision temperature sensing in magnetic resonance imaging (MRI) system applications. 


\section{ACKNOWLEDGEMENT}

The authors would like to thank Eng. Waleed Al Bourihfor his participation throughout this work.

\section{REFERENCES}

[1] A. A. Khan, M. A. Al-Turaigi, A. R. M Alamoud, "Linearized thermistor thermometer using an analog multiplier", IEEE Transactions on Instrumentation and Measurement, Vol. 37, No. 2, pp. 322-323, 1988

[2] S. Pradhan, S. Sen, "An improved lead compensation technique for three-wire resistance temperature detectors", IEEE Transactions on Instrumentation and Measurement, Vol. 48, No. 5, pp. 903-905, 1999

[3] P. R. Nagarajan, B. George, V. J. Kumar, "A linearizing digitizer for wheatstone bridge based signal conditioning of resistive sensors", IEEE Sensors Journal, Vol. 17, No. 6, pp. 1696-1705, 2017

[4] L. Smutny, "Smart Temperature Sensors for Measurement and Control", International Scientific Conference of FME, Session 4: Automation Control and Applied Informatics, Ostrava, Poruba, 2000

[5] S. M. A. Ghaly, S. S. Al-Sowayan, "A high B1 field homogeneity generation using free element elliptical four-coil system", American Journal of Applied Sciences, Vol. 11, No. 4, pp. 534-540, 2014

[6] S. M. A. Ghaly, K. A. Al-Snaie, S. S. Al-Sowayan, "Design and testing of radiofrequency spherical", Modern Applied Science, Vol. 10, No. 5, pp. 186-193, 2016

[7] S. M. A. Ghaly, K. A. Al-Snaie, M. O. Khan, "Spherical and improved helmholtz coil with high B1 homogeneity for magnetic resonance imaging”, American Journal of Applied Sciences, Vol. 13, No. 12, pp. 1413-1418, 2016

[8] S. M. A. Ghaly, K. A. Al-Snaie, A. M. Ali, "Design and modeling of a radiofrequency coil derived from a helmholtz structure", Engineering, Technology \& Applied Science Research, Vol. 9, No. 2, pp. 4037-4043, 2019

[9] Z. P. Nenova, T. G. Nenov, "Linearization circuit of the thermistor connection", IEEE Transactions on Instrumentation and Measurement, Vol. 58, No. 2, pp. 441-449, 2009

[10] A. N. Hosny, I. A. E. Moaty, "Development of a linear temperature measuring circuit using a thermistor", Modelling, Measurement \& Control A, Vol. 63, No. 1-3, pp. 25-30, 1995

[11] C. Renneberg, T. Lehmann, "Analog Circuits for Thermistor Linearization with Chebyshev-Optimal Linearity Error", 18th European Conference on Circuit Theory and Design, Seville, Spain, August 27-30, 2007

[12] Y. J. Wang, L. K. Yang, H. L. Jin, P. Gao, S. J. Zhang, “An Optimal Linearization Method of Parameter Design for NTC Thermister Interface Circuit", International Conference on Mechanics Design, Manufacturing and Automation, 2016

[13] S. K. Sen, T. K. Pan, P. Ghosal, “An improved lead wire compensation technique for conventional four wire resistance temperature detectors (RTDs)", Measurement, Vol. 44, No. 5, pp. 842-846, 2011

[14] S. M. A. Ghaly, "LabVIEW Based Implementation of Resistive Temperature Detector Linearization Techniques", Engineering, Technology \& Applied Science Research, Vol. 9, No. 4, pp. 4530-4533, 2019

[15] F. Reverter, J. Jordana, M. Gasulla, R. P. Areny, "Accuracy and resolution of direct resistive sensor-to-microcontroller interfaces", Sensors and Actuators A: Physical, Vol. 121, No. 1, pp. 78-87, 2005

[16] A. Gani, M. J. E. Salami, "A LabVIEW Based Data Acquisition System for Vibration Monitoring and Analysis", Student Conference on Research and Development, Shah Alam, Malaysia, July 17, 2002

[17] F. Alorifi, S. M. A. Ghaly, M. Y. Shalaby, M. A. Ali, M. O. Khan, "Analysis and detection of a target gas system based on TDLAS \& LabVIEW", Engineering, Technology \& Applied Science Research, Vol. 9, No. 3, pp. 4196-4199, 2019

[18] C. J. Kalkman, "LabVIEW: A software system for data acquisition, data analysis, and instrument control", Journal of Clinical Monitoring, Vol. 11, No. 1, pp. 51-58, 1995 